\title{
Management of the axilla in patients with breast cancer
}

\author{
Amit Goyal
}

Received: 25 November 2009 / Accepted: 3 December 2009

(C) Association of Surgeons of India 2009

\begin{abstract}
This article reviews the changes in management of the axilla in patients with breast cancer in the last decade. It discusses the recent advances, existing controversies and provides evidence-based guidelines for use in clinical practice.

Sentinel lymph node (SLN) biopsy has replaced the more morbid axillary lymph node dissection (ALND) and four node sampling for axillary nodal staging. Blue dye guided four node sampling is an acceptable alternative when radioisotope facilities are not available. ALND is reserved for patients with proven axillary lymph node involvement.

Preoperative axillary ultrasound and fine-needle aspiration cytology or core biopsy of suspicious lymph nodes reliably identifies around $30 \%$ of node positive patients. Intraoperative assessment of the SLN using frozen section or real time molecular assays enables surgeons to perform one stage ALND in node positive patients. For those patients in whom intra-operative SLN assessment is negative, but whose final pathology reveals SLN metastasis, standard treatment has been to perform a completion ALND. Predictive models can be used to identify a lowrisk group of SLN-positive patients in whom routine ALND may not be necessary. In the future, completion ALND for microscopic disease will not be the standard of care but axillary radiotherapy may be an alternative with equal control and less morbidity.
\end{abstract}

\section{A. Goyal}

Department of Surgery,

School of Medicine,

Cardiff University, Cardiff,

CF14 4XN, UK
A. Goyal $(\bowtie)$
E-mail: goyala@cf.ac.uk

Keywords Axillary lymph node dissection - Blue dye · Breast cancer · Four node sampling · Lymphatic mapping · Radioisotope $\cdot$ Sentinel lymph node biopsy

\section{Introduction}

Until recently, the standard approach to the axilla in patients with invasive breast cancer has been axillary lymph node dissection (ALND), which consumes considerable resources and causes both acute and late morbidities for the patient. Complications of ALND include lymphoedema, pain, numbness and limited shoulder movement [1].

\section{Why do patients with invasive breast cancer need to undergo ALND?}

The first reason to perform an ALND is nodal staging. The presence or absence of axillary lymph node involvement is the most important prognostic factor. Adjuvant treatment is advised for all node-positive patients, whereas adjuvant treatment for node-negative patients depends on the presence of other patient and tumour-related factors. The second reason to perform an ALND is to establish the extent of nodal involvement, which might have an impact on adjuvant treatment recommendations. Tumour burden in the axilla may influence the choice of chemotherapy regimen. Patients with 4 or more involved nodes are offered chest wall and supraclavicular fossa radiotherapy to decrease the risk of loco-regional recurrence. The third reason to perform an ALND is to ensure axillary tumour control.

However, management decisions on adjuvant chemotherapy are based increasingly on primary tumour characteristics rather than on nodal status. Nodal status does not change the systemic treatment plan. The incidence 
of axillary metastases has decreased because of earlier detection of the primary tumour by increased use of mammographic screening. Axillary dissection in node negative women exposes them to the complications of this procedure without any benefit. Long-term follow-up data from the NSABP B-04 study suggests that ALND does not confer any significant survival advantage [2]. These developments have prompted exploration of less invasive staging methods for establishing which patients have nodal metastases and therefore need ALND. The most recent overview by the Early Breast Cancer Trialists' Collaborative Group (EBCTCG) revealed that one life is saved at 15 years for every four isolated locoregional recurrences prevented by radiotherapy at 5 years [3]. This implies that local recurrence has a small adverse effect on survival, and regional disease control is important.

\section{Other methods for axillary staging}

Physical examination of the axilla in patients with invasive breast cancer has poor sensitivity and specificity to detect the presence of axillary lymph node metastases. Tumour and patient-related factors are of little value in predicting axillary lymph node involvement in patients with invasive breast cancer. Tumour size has a statistically significant relationship with lymph node metastasis but nevertheless has limited predictive value. Sophisticated techniques such as scintimammography, high-resolution computed tomography (HRCT), dynamic contrast enhanced magnetic resonance imaging (MRI) and positron emission tomography (PET) have been found to be of limited use. The sensitivity and specificity of these methods is inferior to the information from ALND. In addition, early treatment of the axilla in patients with node positive invasive breast cancer ensures excellent regional control and might improve survival in some cases.

\section{Sentinel lymph node biopsy}

Over the past decade, SLN biopsy has become well established as a new standard for axillary node staging in breast cancer. Numerous observational studies of SLN biopsy validated by a completion axillary dissection (ALND) have established that SLN biopsy is feasible, accurate and safe [4]. NEW START SLN biopsy training programme data analyses of more th an 6,000 patients has demonstrated that multiprofessional training and standard methodology can achieve sentinel node identification rate of $>95 \%$ and false negative rate of $<10 \%$ [5]. NICE (National Institute of Clinical Excellence, UK) 2009 guidelines recommend that axillary staging should be performed by SLN biopsy using a combination of isotope and blue dye [6].

\section{Patient selection}

SLN biopsy is suitable for all patients with clinically node negative invasive breast cancer irrespective of tumour size. Many putative "contraindications" (including prior surgical biopsy, large tumour size and multicentricity) have been disproved, but the role of SLN biopsy following neoadjuvant chemotherapy, remains a subject of debate. Patients with T3 tumours have a relatively high-risk of nodal metastases and in the absence of intraoperative sentinel node assessment may be offered ALND at the outset in areas where access to healthcare is limited.

Approximately, $20 \%$ of patients with ductal carcinoma in situ (DCIS) diagnosed by core needle biopsy are upstaged to invasive disease at excision. Presence of a clinically palpable mass or mammographic mass increases the chances of finding an invasive component at excision by approximately 5- to 7-fold. SLN biopsy should be performed at the time of the initial procedure in this subgroup of patients to avoid a second operative procedure for axillary nodal staging [7]. In addition, SLN biopsy should be performed in patients undergoing mastectomy because mastectomy precludes SLN biopsy if invasive disease is subsequently discovered.

\section{Isotope or blue dye}

ALMANAC and NEW START data demonstrates that a combined technique maximises both the success and accuracy of SLN biopsy. The failed localisation rate increases by $3-10 \%$ if only one tracer is used to identify the sentinel node. More importantly, using the blue dye or isotope alone increases the false negative rate by $2-4 \%[4,5]$.

\section{Site and volume of injection}

The breast tissue and its overlying skin drains to the same few axillary SLNs regardless of the injection site. Peritumoural, intradermal, subdermal or subareolar routes of injection have equally good results and a comparably low rate of false negative procedures [8-10]. Peritumoural injection is given in the breast parenchyma at four sites. Intradermal and subdermal injections are given as a single dose into the breast skin directly over the tumour or at the periareolar edge in the tumour quadrant. Subareolar injections are given into the subareolar lymphatic plexus independently of the tumour site. Superficial injection techniques require a much lower-volume of injection than the peritumoural method, and leave a much smaller "hot spot" in the breast. This facilitates the identification of SLN, especially for patients with tumours high in the axillary tail of the breast.

While superficial injections of isotope maximise the 
success of axillary SLN biopsy, they rarely drain to the internal mammary (IM) nodes, which are a well recognised site of lymphatic drainage for the breast. Mapping of the IM nodes requires deep injections, either peritumoural or intratumoural, and demonstrates IM node drainage in $~ 10 \%$ of patients [11]. In contrast to traditional thinking, IM drainage can occur with tumours in any quadrant [11]. IM SLN biopsy is technically demanding and associated with some additional morbidity (pneumothorax, bleeding). IM SLN biopsy is not recommended since $<1 \%$ of all patients will have nodal metastasis isolated to the IM nodes (thereby changing treatment).

Our standard method is to inject the isotope intradermally at a single periareolar site in the tumour quadrant (or cephalad to the previous biopsy scar). Blue dye is injected subdermally at a single periareolar site in the tumour quadrant in the operating room just prior to surgery. The allergic risk of blue dye should be documented and discussed with patients undergoing SLN biopsy when taking consent. Severe allergic reactions are noted in $0.06 \%$ of patients who receive patent blue $\mathrm{V}$ dye [12].

\section{Lymphoscintigraphy}

Preoperative lymphoscintigraphy (LSG) provides a road map for SLN biopsy naïve surgeons but is not needed after the learning phase. LSG identifies the non-axillary SLNs and estimates the number of axillary SLNs (the surgeon should identify at least that many SLNs at operation). The disadvantages are the added time and cost. Since most surgeons are concerned with mapping only to the axilla and majority of patients with a negative LSG will still have "hot" SLN identified intraoperatively by the hand-held gamma probe (a much more sensitive instrument than the gamma camera), many centres have abandoned routine LSG. It may be valuable in patients who have increased risk of intraoperative failed localisation (obese or old patients). A negative preoperative LSG predicts inability to localise with the hand held gamma probe. In patients with a negative LSG, blue dye should be injected intraoperatively to increase the likelihood of SLN identification [13].

LSG may prove particularly useful in those patients who have had previous breast conserving surgery and subsequently develop local recurrence. There is accumulating evidence on the feasibility of "remapping" (SLN biopsy following previous axillary surgery) [14]. Previous axillary operation (SLN biopsy or ALND) changes the lymphatic drainage of the breast unpredictably $[15,16]$. In this setting, LSG identifies a much higher rate of non-axillary lymphatic drainage than observed in "first time" SLN biopsy (30\% versus $6 \%, \mathrm{p}<0.0001$ ), and this information may prove useful in guiding both local and systemic therapy.

\section{Intraoperative assessment of SLN}

Intraoperative assessment of SLN (by frozen section [FS], imprint cytology [IC] or RT-PCR based assays) enables surgeons to perform immediate ALND in SLN-positive patients, avoiding the need for re-operation. However, intraoperative assessment adds to the operative time and cost, and will be falsely negative in some patients. The sensitivity of frozen section is $76 \%$ (95\% C.I. 65-84) and of imprint cytology is $62 \%(95 \%$ CI $53-70)$ [17]. False positives resulting in an unnecessary axillary lymphadenectomy are uncommon with a pooled specificity of $99 \%$. Rapid immunohistochemistry and serial sectioning significantly increase the sensitivity compared to frozen section alone $[18,19]$. However, these techniques have not gained popularity in the UK because of the volume of work involved, time required and the need for a technician and a pathologist to be available at the time of surgery.

Molecular analysis overcomes the sampling error of 'gold standard' histology by assessing the homogenate of the nodal tissue. The GeneSearch ${ }^{\mathrm{TM}}$ BLN Assay is a real time RT-PCR based assay that is performed intraoperatively on fresh homogenised tissue and detects the presence or absence of metastasis in SLNs by quantitatively measuring the expression of mammaglobin and cytokeratin [19]. The assay has been calibrated to detect metastases $>0.2 \mathrm{~mm}$ and has an equivalent sensitivity to serial frozen sections taken every $1.5-2 \mathrm{~mm}[20]$. The assay can be performed and the results interpreted by a trained technician without the need of a pathologist. This allows intraoperative assessment of the SLN in hospitals where immediate pathology is not available due to manpower constraints. The assay has a sensitivity of over $87 \%$ and specificity of over $94 \%$ [21, 22]. The turnaround time of the assay is comparable to that of frozen section ( $\sim 30$ minutes) [22].

\section{ALND when the SLN is positive}

For those patients in whom intraoperative SLN assessment was not done or negative but whose final pathology reveals SLN metastasis, standard treatment has been to perform a completion ALND. This may not be necessary in all cases as in $50 \%$ of patients the SLN is the only positive node [23] and these patients will not benefit from any further axillary surgery. Normograms and predictive models based on factors that predict metastasis to the remaining axillary nodes (the "non-SLN") can be used to estimate the probability of finding non-SLN metastases in SLN positive patients and select patients for ALND $[23,24]$. The ratio of involved to uninvolved nodes is an important determinant of the need for completion ALND. A further possibility is to treat the axilla of patients with a positive SLN with radiotherapy as is being done in the EORTC AMAROS trial [25]. This trial compares regional control of the axilla after delayed ALND 
versus axillary radiotherapy in SLN positive women with invasive breast cancer.

Whether any axillary treatment (surgery or radiotherapy) is needed in patients with metastatic sentinel node disease who receive systemic cytotoxic therapy is also controversial. This question was being addressed by the ACOSOG Z0011 trial in which patients with sentinel node metastases were randomised to completion ALND or no additional axillary therapy. Unfortunately, definitive data from this trial will not be available as it was closed early because of slow accrual and low number of events in both study groups.

\section{Micrometastasis}

The role of immunohistochemical staining of the SLNs and significance of micrometastases remains an area of significant controversy. SLN biopsy and a focused pathologic evaluation results in upstaging of approximately $10-20 \%$ of breast cancer patients. The question is whether this detectable disease is clinically significant. All the present literature is retrospective in nature and the results are inconclusive. Prospective studies are ongoing to evaluate the prognostic significance of micrometastases (IBCSG 23-01 trial, ACOSOG Z0010 trial). Additional H\&E detectable disease within non-SLNs is found in $15-20 \%$ patients with SLN micrometastases (tumour deposits $>0.2 \mathrm{~mm}$ but $\leq 2 \mathrm{~mm}$ ). Therefore, until we have good prospective data, we recommend that completion ALND should be performed in these patients to detect residual H\&E positive non-SLN metastases and plan adjuvant chemotherapy. However, omission of further surgery might be appropriate when micrometastases are present in a single node. Patients with isolated tumour cells (ITCs) on histology should be classified as node negative as there is an emerging consensus that ITCs are of limited prognostic significance.

\section{Axillary ultrasound and fine-needle aspiration or core-needle biopsy}

All patients with clinically node negative invasive breast cancer should routinely undergo a preoperative axillary ultrasound (and FNA or biopsy if indicated) before SLN biopsy. Axillary ultrasound with FNA or core-needle biopsy has a sensitivity of around 30\% [26], but this is dependent on radiologist experience, definition of abnormal node, imaging and biopsy equipment and number of aspiration or biopsy samples taken per axilla. Patients with FNA or biopsy proven metastasis can undergo ALND thus avoiding the unnecessary radiation exposure, morbidity, cost and inconvenience of SLN biopsy. All patients with a normal USS of the axilla or a negative FNA/biopsy of an abnormal node should undergo SLN biopsy because of the high false negative rate.

\section{Multicentric cancer}

It has been disproved that multicentric carcinoma is a contraindication to SLN biopsy. Studies using different injection techniques have demonstrated that all quadrants of the br east drain through common afferent channels to the same axillary sentinel node. The failed localisation and false negative rates of SLN biopsy in women with multicentric disease are comparable to those with unicentric disease [27-29].

\section{Neoadjuvant chemotherapy}

The role of SLN biopsy following neoadjuvant chemotherapy remains a subject of debate. There is no consensus about the timing of SLN biopsy with respect to neoadjuvant chemotherapy in clinically node negative patients. Prechemotherapy SLN biopsy accurately stages the axilla and may affect the choice of chemotherapy regimens used in SLN positive patients. Postchemotherapy SLN biopsy avoids a second surgery and does not delay the start of neoadjuvant chemotherapy. It identifies node positive patients who have been downstaged to N0 by neoadjuvant chemotherapy and therefore can be spared ALND.

On the other hand, this loss of prognostic information may complicate clinical decision making for local treatment, such as whether radiation is indicated after mastectomy, or what regions should be irradiated after lumpectomy. A metaanalysis of 21 studies (1,273 patient with operable breast cancer) showed that SLN biopsy following neoadjuvant chemotherapy had a slightly higher failed localisation rate $(10 \%)$ and the false negative rate (12\%) [30]. There is a concern that neoadjuvant therapy may achieve a 'patchy' kill of metastatic disease in the axillary lymph nodes leading to a higher false negative rate. Therefore, SLN biopsy should be considered before primary systemic therapy, with ALND performed after chemotherapy if disease is present in the SLN.

\section{Reoperative sentinel node biopsy}

Recent published data suggests that SLN "remapping" is feasible in patients who develop ipsilateral breast recurrence or second primary cancer following breast conservation and SLN biopsy. SLN was successfully identified in 74-97\% patients $[15,16,31,32]$. In these studies "remapping" was performed on a small number of patients and information on 
false negative rate is lacking. This is a cause for concern, as the NSABP-32 data has shown that prior excisional biopsy significantly increases the false negative rate (FNA/core $8.0 \%$ versus excisional biopsy $15.2 \%, p=0.02$ ) while not affecting the failed localisation rate. More data is needed before SLN biopsy can be recommended in this setting.

\section{Multiple sentinel lymph nodes}

More than one SLN is present in a majority of patients, and in our own experience identification of multiple SLNs when present is important to reduce the false negative rate [33]. Multiple SLNs represent migration of tracer from the 'true' SLN into secondary echelon nodes, or normal anatomic variation in which the lymphatics of a given site drain simultaneously to more than one SLN. The surgeon is able to identify $99 \%$ or more of node positive patients if he stops after sampling 4 nodes [33]. Removing more than 4 nodes leads to minimal improvement in accuracy while potentially adding to the morbidity of the procedure.

\section{Morbidity}

Data from randomised controlled trials conclusively demonstrates a marked diminution of complications associated with SLN biopsy when compared with ALND [1, 34-36]. SLN biopsy reduces but does not completely eliminate the risk of lymphoedema. The ALMANAC trial compared morbidity of SLN biopsy with that associated with conventional ALND [1]. Analysis at 12 months showed that SLN biopsy was associated with less lymphoedema, sensory deficits, and infections compared with ALND. Quality-of-life was found to be superior and arm-related morbidity was lower for patients who had SLN biopsy.

\section{Local recurrence and survival}

It is premature to make a definitive comment on local recurrence rate or survival following SLN biopsy. These questions will be answered by the ongoing National Surgical Adjuvant Bowel and Breast Project (NSABP-32) and the American College of Surgeons Oncology Group (ACOSOG) trials. A recent meta-analysis of 48 observational studies (14,959 patients) suggests that SLN biopsy is associated with a $<1 \%$ local recurrence rate after a median follow-up of 34 months [37]. The follow-up is relatively short but the results are reassuring as we know from NSABP B-04 that most axillary recurrences in patients treated without ALND occur in the first 2 years [2].

\section{Four node axillary sampling}

Axillary node sampling is a procedure to stage the axilla which has been used in some UK institutions. Four node sampling has been compared with axillary clearance in small randomised studies and has been shown to be more than $95 \%$ accurate in staging the axilla $[38,39]$. It has been criticised as it is blind and relies on palpation to remove four lymph nodes. The technique of axillary sampling has been difficult to standardise and is non-targeted. By contrast, SLN biopsy is a targeted form of sampling that aims to remove the most biologically significant nodes. Sensory loss and lymphoedema are significantly worse after four node sampling compared with sentinel node biopsy [40].

\section{Blue dye guided four node sampling}

This technique represents blue dye targeted four node sampling. The blue SLNs are harvested first and then other palpable nodes usually from level 1 are identified and removed. The aim is to remove a total of at least four lymph nodes. This allows accurate staging of the axilla while limiting surgical dissection and morbidity of the procedure [41]. There are no data directly comparing arm morbidity in patients undergoing blue dye assisted sampling with those having SLN biopsy. The use of radioisotope presents financial and logistical problems for some breast cancer units. Blue dye guided four node sampling could be a more pragmatic and cheaper approach for these centres around the world.

\section{Summary}

Patients with invasive breast cancer and selected patients with DCIS (presence of clinical or mammographic mass, patients undergoing mastectomy) should undergo axillary nodal staging by SLN biopsy using both radioisotope and blue dye. The final step in SLN biopsy (after removal of all blue and/or hot SLN) is careful palpation of the axilla, and the removal of any palpably suspicious nodes to reduce the false negative rate. Preoperative axillary ultrasound with FNA or core biopsy of suspicious nodes should be performed on all patients to identify axillary metastases and therefore, allow ALND at the outset without preliminary SLN biopsy.

Single tracer methods yield inferior results (identification rate and false negative rate) compared with the combined technique (isotope and blue dye). Blue dye guided four node sampling is an acceptable method for staging the axilla and is pragmatic and cost-effective when radioisotope facilities are not available. Completion ALND 
is the preferred method for treating all patients with either macrometastatic or micrometastatic deposits in the SLN. Omission of further axillary surgery is acceptable for ITCs, presence of micrometastasis in a single SLN and patients with a predicted low risk for non-SLN involvement. In the future, completion ALND for microscopic disease will not be the standard of care but axillary radiotherapy may be an alternative with equal control and less morbidity.

\section{Reference}

1. Mansel RE, et al. (2006) Randomised multicenter trial of sentinel node biopsy versus standard axillary treatment in operable breast cancer: the ALMANAC Trial. J Natl Cancer Inst 98:599-609

2. Fisher B, et al. (2002) Twenty-five-year follow-up of a randomised trial comparing radical mastectomy, total mastectomy, and total mastectomy followed by irradiation. N Engl J Med 347:567-575

3. Clarke M, et al. (2005) Effects of radiotherapy and of differences in the extent of surgery for early breast cancer on local recurrence and 15-year survival: an overview of the randomised trials. Lancet 366:2087-2106

4. Goyal A, Newcombe RG, Chhabra A, Mansel RE (2006) Factors affecting failed localisation and false-negative rates of sentinel node biopsy in breast cancer--results of the ALMANAC validation phase. Breast Cancer Res Treat 99:203-208

5. Goyal A, et al. Results of the UK NEW START sentinel node biopsy training program: a model for future surgical training. Proceedings of 32nd annual San Antonio Breast Cancer Symposium 2009.

6. http://www.nice.org.uk/Guidance/CG80. Accessibility checked on 28/02/09 2009 .

7. Goyal A, et al. (2006) Is there a role of sentinel lymph node biopsy in ductal carcinoma in situ? Analysis of 587 cases. Breast Cancer Res Treat 98:311-314

8. Povoski SP, et al. (2006) Prospective randomised clinical trial comparing intradermal, intraparenchymal, and subareolar injection routes for sentinel lymph node mapping and biopsy in breast cancer. Ann Surg Oncol 13:1412-1421

9. McMasters KM, et al. (2001) Dermal injection of radioactive colloid is superior to peritumoral injection for breast cancer sentinel lymph node biopsy: results of a multiinstitutional study. Ann Surg 233:676-687

10. Klimberg VS, et al. (1999) Subareolar versus peritumoral injection for location of the sentinel lymph node. Ann Surg 229:860-864

11. Mansel RE, Goyal A, Newcombe RG (2004) Internal mammary node drainage and its role in sentinel lymph node biopsy: the initial ALMANAC experience. Clin Breast Cancer 5:279-284

12. Barthelmes L, Goyal A, Newcombe RG, McNeill F, Mansel RE (2009) Adverse reactions to patent blue V dye - The NEW START and ALMANAC experience. Eur J Surg Oncol (In press)

13. Goyal A, et al. (2005) Role of routine preoperative lymphoscintigraphy in sentinel node biopsy for breast cancer. Eur J Cancer 41:238-243
14. Port ER, et al. (2002 ) Reoperative sentinel lymph node biopsy: a new option for patients with primary or locally recurrent breast carcinoma. J Am Coll Surg 195:167-172

15. Port ER, et al. (2007) Reoperative sentinel lymph node biopsy: a new frontier in the management of ipsilateral breast tumor recurrence. Ann Surg Oncol 14:2209-2214

16. Intra $M$, et al. (2007) Second axillary sentinel node biopsy for ipsilateral breast tumour recurrence. Br J Surg 94:1216-1219

17. Tew K, Irwig L, Matthews A, Crowe P, Macaskill P (2005) Meta-analysis of sentinel node imprint cytology in breast cancer. Br J Surg 92:1068-1080

18. Johnston EI, Beach RA, Waldrop SM, Lawson D, Cohen C (2006) Rapid intraoperative immunohistochemical evaluation of sentinel lymph nodes for metastatic breast carcinoma. Appl Immunohistochem Mol Morphol 14:57-62

19. Viale G, et al. (1999) Intraoperative examination of axillary sentinel lymph nodes in breast carcinoma patients. Cancer 85: 2433-2438

20. Viale G, et al. (2008) Comparative evaluation of an extensive histopathologic examination and a real-time reverse-transcription-polymerase chain reaction assay for mammaglobin and cytokeratin 19 on axillary sentinel lymph nodes of breast carcinoma patients. Ann Surg 247:136-142

21. Blumencranz P, et al. (2007) Scientific Impact Recognition Award. Sentinel node staging for breast cancer: intraoperative molecular pathology overcomes conventional histologic sampling errors. Am J Surg 194:426-432

22. Mansel RE, et al. (2008) Detection of breast cancer metastasis in sentinel lymph nodes using intra-operative real time GeneSearchtrade mark BLN Assay in the operating room: results of the Cardiff study. Breast Cancer Res Treat

23. Goyal A, Douglas-Jones A, Newcombe RG, Mansel RE (2004) Predictors of non-sentinel lymph node metastasis in breast cancer patients. Eur J Cancer 40:1731-1737

24. Park J, et al. (2007) A declining rate of completion axillary dissection in sentinel lymph node-positive breast cancer patients is associated with the use of a multivariate nomogram. Ann Surg 245:462-468

25. Mansel RE, Goyal A (2004) European studies on breast lymphatic mapping. Semin Oncol 31:304-310

26. Baruah B, Goyal A, Young P, Douglas-Jones A, Mansel R (2009) Axillary nodal staging by ultrasonography and fineneedle aspiration cytology in patients with breast cancer. Br J Surg (in press)

27. Goyal A, et al. (2004) Sentinel lymph node biopsy in patients with multifocal breast cancer. Eur J Surg Oncol 30:475-479

28. Schrenk P, Wayand W (2001) Sentinel-node biopsy in axillary lymph-node staging for patients with multicentric breast cancer. Lancet 357:122

29. Knauer M, et al. (2006) Multicentric breast cancer: a new indication for sentinel node biopsy--a multi-institutional validation study. J Clin Oncol 24:3374-3380

30. Xing Y, et al. (2006) Meta-analysis of sentinel lymph node biopsy after preoperative chemotherapy in patients with breast cancer. Br J Surg 93:539-546

31. Taback B, et al. (2006) Sentinel lymph node biopsy for local recurrence of breast cancer after breast-conserving therapy. Ann Surg Oncol 13:1099-1104 
32. Cox CE, et al. (2008) Use of reoperative sentinel lymph node biopsy in breast cancer patients. J Am Coll Surg 207:57-61

33. Goyal A, Newcombe RG, Mansel RE (2005) Clinical relevance of multiple sentinel nodes in patients with breast cancer. Br J Surg 92:438-442

34. Purushotham AD, et al. (2005) Morbidity after sentinel lymph node biopsy in primary breast cancer: results from a randomised controlled trial. J Clin Oncol 23:4312-4321

35. Veronesi U, et al. (2003) A randomised comparison of sentinel-node biopsy with routine axillary dissection in breast cancer. N Engl J Med 349:546-553

36. Zavagno G, et al. (2008) A Randomised clinical trial on sentinel lymph node biopsy versus axillary lymph node dissection in breast cancer: results of the Sentinella/GIVOM trial. Ann Surg 247:207-213

37. van der Ploeg IM, Nieweg OE, van Rijk MC, Valdés Olmos RA, Kroon BB. (2008) Axillary recurrence after a tumournegative sentinel node biopsy in breast cancer patients:
A systematic review and meta-analysis of the literature. Eur J Surg Oncol 34(12):1277-1284

38. Forrest AP, et al. (1995) The Edinburgh randomised trial of axillary sampling or clearance after mastectomy. Br J Surg 82:1504-1508

39. Chetty U, Jack W, Prescott RJ, Tyler C, Rodger A (2000) Management of the axilla in operable breast cancer treated by breast conservation: a randomised clinical trial. Edinburgh Breast Unit. Br J Surg 87:163-169

40. Monypenny IJ, Dixon M, Kissin M, Newcombe RG, Goyal A, Mansel RE. (2005) End of 4 node sampling?- Comparative morbidity versus sentinel lymph node biopsy in the ALMANAC trial. Proceedings of the Nottingham Breast Cancer Conference 2005.

41. Chetty U, Chin PK, Soon PH, Jack W, Thomas JS (2008) Combination blue dye sentinel lymph node biopsy and axillary node sampling: the Edinburgh experience. Eur J Surg Oncol 34:13-16 